\title{
The optimization of automated goods dynamic allocation and warehousing model
}

\author{
Z.K.Hou ${ }^{l}$

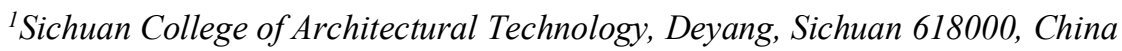

\begin{abstract}
In the development of modern logistics, the role of automated cargo warehousing is gradually reflected, which is essential for the automatic distribution of goods. This paper briefly introduced the automatic location allocation model and the particle swarm optimization (PSO) algorithm used to optimize the model. At the same time, it introduced the concept of genetic operator and multigroup co-evolution to improve the algorithm, and then the simulation analysis of standard PSO and improved PSO was performed on MATLAB software. The results showed that the improved PSO iterated fewer times and get better solution sets; compared with the manual allocation scheme, the improved PSO calculation reduced more warehousing time, lowered more center of gravity height, and improved shelf stability. In summary, the improved PSO algorithm can effectively optimize the automated goods dynamic allocation and warehousing model.

Keywords: location allocation, particle swarm optimization, genetic operator, multi-group co-evolution.

Citation: Hou ZK. The optimization of automated goods dynamic allocation and warehousing model. Computer Optics 2020; 44(5): 843-847. DOI: 10.18287/2412-6179-CO-682.

Acknowledgments: This study was supported by 2016 Special Task of Scientific and Technological Research in Sichuan College of Architectural Technology: Research and Design on Small Automatic Sorting and Accessing Stereo Warehouse in University Jingdong Delivery Based on Jingdong Small Parcel Logistics Data (2016KJ36).
\end{abstract}

\section{Introduction}

Logistics is defined as the collection of a series of work such as packaging, storage, and distribution of goods during the process of transporting goods from the seller to the buyer [1]. In modern logistics, in order to improve efficiency, automated stereoscopic warehouse is generally adopted to realize automatic distribution of goods [2]. In this process, different kinds of goods continue to circulate the process of "loading-storageunloading". After a period of time, the stacking of goods on the shelves will become disorderly, which will not only affect the efficiency of storage, but also affect the stability of tridimensional shelves [3]. Therefore, real-time optimization of the cargo location allocation is required. When allocating cargo space, it is not only necessary to maintain the overall stability of the shelf, but also to ensure the utilization of storage space and the efficiency of loading and unloading. Common algorithms include enumeration method, random method and evolutionary algorithm [4]. The optimized location allocation model can effectively improve logistics efficiency and enhance shelves stability. Relevant studies are as follows. Fontana et al. [5] proposed a multi-criteria decision-making model to classify and store products in multi-storey warehouse and solve it. The simulation results showed that the method could effectively improve the order response efficiency. Simulation results showed that the method could effectively improve order response efficiency. Dijkstra et al. [6] used the path length formula and the optimality attribute to determine the allocation of goods storage loca- tion in order to improve the efficiency of goods in and out of warehouse. The experimental results showed that the method could improve the efficiency of goods in and out of warehouse. Xie et al. [7] proposed a new two-layer grouping optimization model for the location allocation with grouping constraints and solved the model using the multi-stage random search method and the tabu algorithm. The experimental results showed the effectiveness of the model and algorithm. This paper briefly introduced the automatic cargo space allocation model and the particle swarm optimization (PSO) algorithm used to optimize the model, and improved the algorithm by introducing the concepts of genetic operator and multi-population coevolution. Then the simulation analysis of standard PSO and improved PSO was carried out in MATLAB software. In this study, the optimal scheme of automatic distribution and warehousing of goods was calculated using the PSO algorithm, so as to improve the efficiency of logistics. The novelty of this study is that genetic algorithm is introduced into the standard PSO algorithm to make PSO algorithm avoid the defect of premature in the iterative process, so as to further improve the efficiency and optimization results of the algorithm.

\section{Automated cargo space allocation model}

In the paper, some assumptions are made for the convenience of explanation and calculation when establishing the warehousing model of goods distribution: (1) the goods are distributed through a single entrance and exit; (2) the relevant information of the goods in storage is known, and the total amount of it cannot exceed that of 
the warehouse; (3) The spacing between the shelves is the same as the width of the shelves; (4) the same kind of goods is regarded as one cargo, and only one cargo is stored in one cargo space; (5) when goods are put into storage, the working speed of the automatic tools used for transportation remains unchanged, and only one cargo is transported at a time.

There are two objectives of the goods distribution warehousing model, that is, to place the light goods at the top and the heavy good at the bottom as far as possible and to get the goods in and out of the warehouse as efficiently as possible. The function formula used to describe the optimization effect of the previous objective is:

$$
f_{1}(x, y, z)=\sum_{x=1}^{n} \sum_{y=1}^{p} \sum_{z=1}^{q} m_{x y z} y A_{x y z},
$$

where $f_{1}$ stands for the function of the center of gravity of the shelves, the smaller the function, the higher the stability; $x, y, z$ represents the number of shelf rows, the number of layers, and the number of columns; $n, p, q$ represents the maximum number of rows, the maximum number of layers, and the maximum number of columns in the shelf; $m_{x y z}$ represents the mass of the goods in the row $x$ layer $y$ column $z ; A_{x y z}$ represents whether there are any goods in row $x$ layer $y$ column $z, 1$ if there are and 0 if not.

The function formula [8] used to describe the efficiency of warehousing is:

$$
\left\{\begin{array}{l}
f_{2}(x, y, z)=\sum_{x=1}^{n} \sum_{y=1}^{p} \sum_{z=1}^{q} f_{x y z} T_{x y z} \\
T_{x y z}=t_{x}+t_{y}+t_{z}
\end{array},\right.
$$

where $f_{2}$ is a function of warehousing efficiency, following the principle of proximity; $f_{\mathrm{xyz}}$ represents the access frequency in $(x, y, z)$ coordinates; $T_{x y z}$ represents the total time required by the automatic transportation equipment to store the goods in the cargo space of row $x$ layer $y$ column $z ; t_{x}, t_{y}, t_{z}$ represents the time required for automatic transport equipment to transport goods to row $x$, layer $y$ and column $z$.

In summary, the mathematical expression of automatic cargo space allocation model [9] is:

Objective function:

$$
\begin{aligned}
& f(x, y, z)=\alpha f_{1}(x, y, z)+\beta f_{2}(x, y, z) \Rightarrow f_{\min }, \\
& \text { condition: }\left\{\begin{array}{l}
x \in[1, n] \\
y \in[1, p] \\
z \in[1, q]
\end{array}\right.
\end{aligned}
$$

where $\alpha, \beta$ are the proportion weights of $f_{1}$ and $f_{2}$ in the whole model.

\section{Improved PSO}

PSO, whose full name is particle swarm algorithm, is one of the evolutionary algorithms, and the iterative formula [10] is:

$$
\left\{\begin{array}{l}
V_{i+1}=V_{i}+a_{1} \cdot x_{1} \cdot\left(\text { pbest }_{i}-P_{i}\right)+a_{2} \cdot x_{2} \cdot\left(\text { gbest }_{i}-P_{i}\right) \\
P_{i+1}=P_{i}+V_{i+1}
\end{array},\right.
$$

where $P_{i}, V_{i}$ are the position and velocity of particle $\mathrm{i}$; pbest $_{i}$, gbest ${ }_{i}$ are the individual optimal position and the global optimal position respectively; $a_{1}, a_{2}$ are the learning factors; $x_{1}, x_{2}$ are the random numbers which evenly distribute between 0 and 1 . The iteration stops when the optimal solution is found or the maximum number of iterations is reached. The PSO iteration formula is as described above. It is seen from the formula that the principle of the algorithm is relatively simple: the position is randomly searched using a large population of particles in the search space first, then the relatively appropriate position is found out, and the rest of the particles will gather to the appropriate position, just like the rest of the birds follow the leader when migrating. According to the above PSO principle analysis, it can be seen that pbest $t_{i}$ and gbest $_{i}$ play a very important role in the iteration process which applies the iteration formula, the former is the individual optimal position, and the latter is the global optimal position. The individual optimal position refers to the optimal position searched by a single individual particle $i$ in the particle population in the iteration process, and the global optimal position is the position of the particle with the optimal fitness in the population in the iteration.

In order to overcome the shortcoming of the abovementioned standard PSO algorithm, which is easy to "premature", this paper introduces genetic operators and implements multi-group co-evolution. The flow of the improved PSO algorithm is shown in Fig. 1.

1. Relevant parameters were imputed, including particle swarm size, crossover, mutation probability, learning factor and maximum number of iterations, etc., and then multiple initial populations were generated. Three initial populations are selected in this paper. Each particle $X_{i}$ in the population represents a kind of cargo warehousing plan, which is encoded as: $X_{i}=\left(X_{i 1}, X_{i 2}, \cdots, X_{i m}, \cdots, X_{i D},.\right)$, where $X_{i m}=\left(x_{i m}, y_{i m}, z_{i m}\right)$ indicates the cargo position of item $\mathrm{m}$ in the cargo warehousing plan i, and $m \in(1,2,3, \cdots, D), D$ indicates the number of goods species. The particles in the initial population are randomly generated, the particles containing duplicate cargo positions are removed before participating in the iteration, and new random particles are added to meet the population size requirements.

2. The fitness values of the particles in each population were calculated, and the individual optimal solution and the global optimal solution of each population were selected. The adaptive function is equation (3) above. The fitness value is the value reflecting the excellent degree of the solution of particle search, which is calculated by the fitness function formula. The fitness function which is used for calculating the fitness value in this paper is equation (3) mentioned above, i.e., the objective function, because the purpose of using PSO algorithm is to search out the best scheme of goods distribution and warehousing, and the method which is used for measuring the quality 
of the scheme is calculating the comprehensive values of center of gravity of shelf and warehousing efficiency with equation (3) (the smaller the values, the more excellent the scheme). The individual optimal solution and global optimal solution are the particle individual optimal posi- tion and the global optimal position mentioned in the above description of PSO iteration formula. In the PSO algorithm, a particle represents a distribution scheme, and its position in the search space is the content of the corresponding distribution scheme.

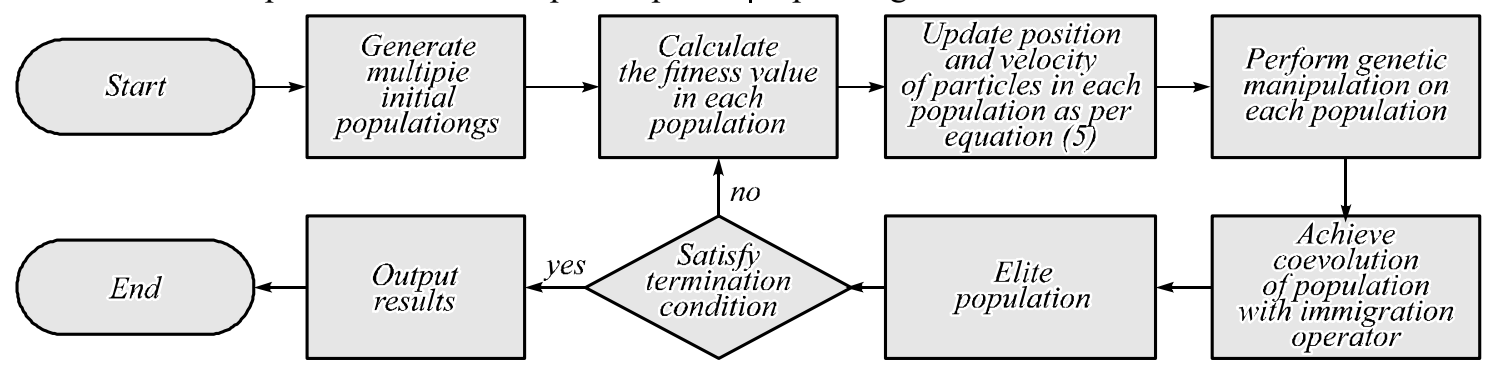

Fig. 1. The flow of the improved PSO algorithm

3 . The position and velocity of the particles according to equation (5) in each population were updated and participated in the next step.

4. The particles in each population is genetically manipulated, including crossover and variation [11]. The crossover operation uses a single-point crossover operator, that is, selecting two particles that reach the crossover probability, randomly selecting one gene position from them, and then exchanging the coordinates of the cargo position in the gene position to form two new particles; the mutation operation adopts the basic mutation operator. When the gene position in the particle reaches the mutation probability, the coordinate of the cargo position in the gene position randomly become the cargo coordinates that are not repeated with other gene positions of the same particle and are within the limit range.

5. After the end of each population's genetic operation, the migration operator [12] is used to make the three populations communicate and achieve the effect of coevolution. The main operation is to replace the worst particles of the next population with the best particle of the population in a sequential cycle.

6 . The best particles in the population were selected from the current number of iterations; and were copied and stored in an independent elite population, which does not participate in the multi-population evolutionary iteration. After storing the optimal particles in the contemporary population into the elite population, the other three populations are determined whether to continue the iteration or not according to the termination conditions.

7. The termination condition is generally that the maximum number of iterations was reached or the fitness converged to stability. In addition to the maximum number of iterations, the termination condition of this paper also determined whether the algorithm continued to iterate according to the optimal particle retention algebra in elite population [13]. Each iteration of the algorithm stored the best contemporary particles into the elite population. When the optimal particles stored in each time remain were kept unchanged, and the continuous algebra reached the set minimum maintenance algebra, the iteration stopped. Then the optimal particle in the elite popu- lation was output to obtain the optimal solution set of the allocation scheme.

\section{Experimental analysis}

\section{Experimental environment}

This paper used MATLAB software [14] to simulate and analyze the standard PSO algorithm and the improved PSO algorithm. The experiment was carried out on a laboratory server. The operating system was Windows 7, the CPU was Core I7, and the memory size was $16 \mathrm{G}$.

2. Experimental parameters

Table. 1. Access frequency and mass of goods

\begin{tabular}{|l|l|l|l|l|l|}
\hline $\begin{array}{l}\text { Prod- } \\
\text { uct } \\
\text { num- } \\
\text { ber }\end{array}$ & $\begin{array}{l}\text { Access } \\
\text { fre- } \\
\text { quenc } \\
\mathrm{y}\end{array}$ & $\begin{array}{l}\text { Mass/ } \\
\mathrm{kg}\end{array}$ & $\begin{array}{l}\text { Prod- } \\
\text { uct } \\
\text { num- } \\
\text { ber }\end{array}$ & $\begin{array}{l}\text { Access } \\
\text { frequen- } \\
\text { cy }\end{array}$ & $\begin{array}{l}\text { Mass/k } \\
\mathrm{g}\end{array}$ \\
\hline 1 & 0.24 & 35 & 6 & 0.55 & 56 \\
\hline 2 & 0.58 & 45 & 7 & 0.20 & 15 \\
\hline 3 & 0.66 & 55 & 8 & 0.32 & 20 \\
\hline 4 & 0.41 & 60 & 9 & 0.31 & 25 \\
\hline 5 & 0.61 & 40 & 10 & 0.33 & 35 \\
\hline
\end{tabular}

The access frequency and mass of the goods are shown in Table. 1. The relevant parameters of the autostereoscopic shelf were: row 5 , layer 5 and column 6 ; the length, width and height of the SKU were $1 \mathrm{~m}$; the speed of automatic transport equipment was $1.5 \mathrm{~m} / \mathrm{s}$ horizontally and $1 \mathrm{~m} / \mathrm{s}$ vertically.

The parameters of the standard PSO algorithm were: the population size was 30 ; the learning factor $a_{1}, a_{2}$ were 2.0; the maximum number of iterations was 400 .

The parameters of the improved PSO algorithm were: the size, learning factor and maximum number of iterations of the three populations were the same as the standard PSO algorithm; the crossover probability of each population was 0.8 , and the mutation probability was 0.0008 .

3. Field experiment

In order to further verify the effectiveness of the improved PSO algorithm, the traditional PSO algorithm and improved PSO algorithm were applied to the actual au- 
tomatic warehouse allocation. In the field experiment, there were 7 rows, 6 layers and 7 columns of automatic three-dimensional shelves, and the length, width and height of each compartment in the three-dimensional shelves were $1.2 \mathrm{~m}, 1.1 \mathrm{~m}$ and $1.3 \mathrm{~m}$ respectively. Moreover, the horizontal speed and vertical movement speed of the automatic transportation equipment matched with the automatic three-dimensional shelves were $1.4 \mathrm{~m} / \mathrm{s}$ and $1.2 \mathrm{~m} / \mathrm{s}$ respectively.

The scale of the goods for this field experiment was 150 pieces, and the specifications of single goods did not exceed the specifications of the shelf; the access frequency was between 0.2 and 0.6 , and the mass of single goods was between $35 \mathrm{~kg}$ and $70 \mathrm{~kg}$. Due to the large quantity of goods, limited by the length, the detailed information of single goods is not listed here.

The standard PSO algorithm and improved PSO algorithm which were used for calculating the automatic cargo distribution scheme were compiled by MATLAB software, and the relevant parameters were consistent with the simulation experiment above. Moreover, skilled employees with 3 years of work experience were assigned manually to obtain the allocation scheme, and the scheme was compared with the allocation scheme obtained by the first two algorithms.

4. Experimental results

After 400 times of iterations, the fitness of the population in the two algorithms converged gradually. The convergence curve is shown in Fig. 2. It can be seen from the Fig. that the standard PSO algorithm converged to stability after about 50 times of iterations, and the improved PSO algorithm converged to stability only after about 20 times of iterations. It can be clearly seen that the optimal solution obtained by the improved PSO algorithm was better when it converged stably, which also indicated that the standard PSO algorithm fell into the phenomenon of "premature" when the convergence was stable, and the optimal solution found when it converged to stability was not the true global optimal solution.

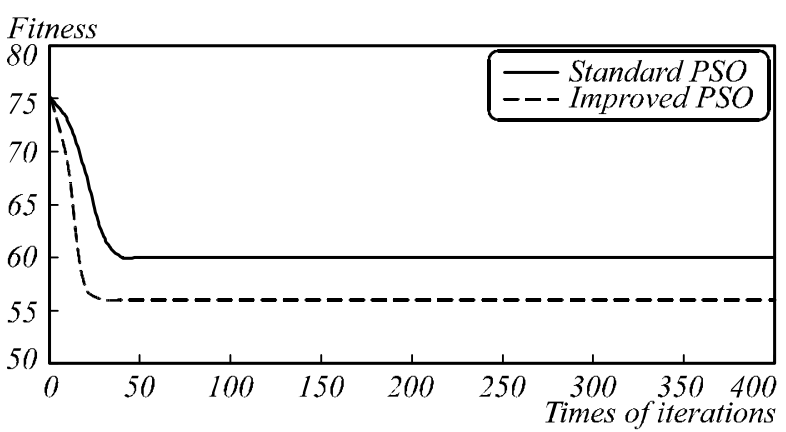

Fig. 2. Convergence curves of the two algorithms

The warehousing time and the overall height of center of gravity obtained by the two-segment optimized location allocation scheme are shown in Table 2. At the same time, in order to illustrate the optimization effect of the two algorithms on the location allocation model, the allocation schemes calculated by the two algorithms were compared with the traditional manual allocation scheme. Among them, the traditional manual allocation scheme required $3654 \mathrm{~s}$ to allocate goods into warehouse, the standard PSO algorithm required $1290 \mathrm{~s}$, which was reduced by $64.70 \%$, and the improved PSO algorithm required $1130 \mathrm{~s}$, which was reduced by $69.07 \%$. In the traditional manual allocation scheme, the overall height of center of gravity of the three-dimensional shelf was 4.123 $\mathrm{m}$. The overall height of center of gravity of the standard PSO calculation based scheme was $3.847 \mathrm{~m}$, showing a decrease of $6.69 \%$, and the overall height of center of gravity of the improved PSO calculation based scheme was $3.543 \mathrm{~m}$, which decreased by $14.07 \%$. It can be seen that the cargo allocation based scheme optimized by the optimized PSO algorithm had a shorter storage time and a lower height of center of gravity, which means the threedimensional shelf is more stable. The optimization effect of the improved PSO algorithm was better.

Table. 2. Optimization effects of two algorithms on automatic cargo allocation model

\begin{tabular}{|l|l|l|l|}
\hline & $\begin{array}{l}\text { Artificial } \\
\text { alloca- } \\
\text { tion }\end{array}$ & $\begin{array}{l}\text { Standard } \\
\text { PSO algo- } \\
\text { rithm }\end{array}$ & $\begin{array}{l}\text { Improved } \\
\text { PSO } \\
\text { algorithm }\end{array}$ \\
\hline $\begin{array}{l}\text { Allocation } \\
\text { and ware- } \\
\text { housing } \\
\text { time / s }\end{array}$ & 3654 & 1290 & 1130 \\
\hline $\begin{array}{l}\text { Optimization } \\
\text { effect com- } \\
\text { pared to arti- } \\
\text { ficial alloca- } \\
\text { tion }\end{array}$ & $/$ & $64.70 \%$ & $69.07 \%$ \\
\hline $\begin{array}{l}\text { Height of cen- } \\
\text { ter of gravi- } \\
\text { ty/m }\end{array}$ & 4.123 & 3.847 & 3.543 \\
\hline $\begin{array}{l}\text { Optimization } \\
\text { effect com- } \\
\text { pared to arti- } \\
\text { ficial alloca- } \\
\text { tion }\end{array}$ & $/$ & $6.69 \%$ & $14.07 \%$ \\
\hline
\end{tabular}

The allocation and warehousing time and height of center of gravity of the distribution scheme optimized by the two algorithms and the scheme obtained by manual distribution are shown in Fig. 3. In the scheme of manual distribution, the warehousing of goods needed $13250 \mathrm{~s}$; in the scheme obtained by the standard PSO algorithm, the warehousing of goods needed $10250 \mathrm{~s}$; in the scheme obtained by the improved PSO algorithm, $8890 \mathrm{~s}$ was needed. The height of center of gravity of goods was $5.325 \mathrm{~m}$ in the scheme of manual distribution, $4.123 \mathrm{~m}$ in the scheme obtained by the standard PSO algorithm, and $2.894 \mathrm{~m}$ in the scheme obtained by the improved PSO algorithm. It was seen from Fig. 3 that the distribution scheme obtained by the PSO algorithm had more advantages than the manual distribution method in the efficiency of distribution and warehousing and the stability of center of gravity of goods 
in the field experiment, and the improved PSO algorithm obtained a distribution scheme with higher warehousing efficiency and more stable center of gravity of good than the standard PSO algorithm.

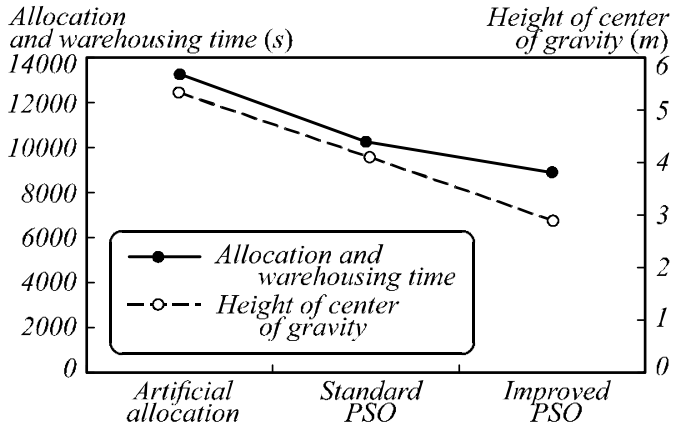

Fig. 3. The optimization effects of the distributions schemes obtained by the two algorithms in the field experiment

\section{Conclusion}

This paper briefly introduced the automatic location allocation model and the PSO algorithm used to optimize the model. At the same time, it introduced the concept of genetic operator and multi-group co-evolution to improve the algorithm, then the simulation analysis of standard PSO and improved PSO algorithm was performed on MATLAB software. The results are as follows: (1) the standard PSO algorithm converged to stability after about 50 times of iteration, and the improved PSO algorithm converged to stability after about 20 times of iteration; the fitness after stabilization was smaller than the standard PSO algorithm, which was more excellent; (2) compared with the manual allocation scheme, the allocation scheme obtained by the standard PSO algorithm reduced the storage time by $64.70 \%$ and the shelf gravity center by $6.69 \%$; the scheme obtained by the improved PSO algorithm reduced the storage time by $69.07 \%$ and the shelf gravity center by $14.07 \%$, so the optimization effect of improved PSO algorithm was better; (3) the results of the field experiment further verified that the PSO algorithm could obtain a distribution scheme with better warehousing efficiency and more stable center of gravity of goods and that the distribution scheme obtained by the improved PSO algorithm had higher warehousing efficiency and more stable center of gravity.

\section{References}

[1] Petering M. Real-time container storage location assignment at an RTG-based seaport container transshipment terminal: problem description, control system, simulation model, and penalty scheme experimentation. Flexible Services \& Manufacturing Journal 2015; 27(2-3): 351-381.

[2] Yang CL, Nguyen TPQ. Constrained clustering method for class-based storage location assignment in warehouse. Industrial Management \& Data Systems 2016; 116(4): 667-689.

[3] Saboori H, Hemmati R. Optimal management and planning of storage systems based on particle swarm optimization technique. Journal of Renewable \& Sustainable Energy 2016; 8(2): 453-475.

[4] Tsamis N, Giannikas V, Mcfarlane D, Lu W, Strachan J. Adaptive storage location assignment for warehouses using intelligent products. Nature Communications 2015; 6(1): 271-279.

[5] Fontana ME, Nepomuceno VS. Multi-criteria approach for products classification and their storage location assignment. International Journal of Advanced Manufacturing Technology 2016; 88(9-12): 1-12.

[6] Dijkstra AS, Roodbergen KJ. Exact route-length formulas and a storage location assignment heuristic for picker-toparts warehouses. Transportation Research Part E Logistics \& Transportation Review 2017; 102: 38-59.

[7] Xie J, Mei Y, Ernst A T, Li X, Song A. A Bi-level optimization model for grouping constrained storage location assignment problems. IEEE Transactions on Cybernetics 2017; 1-14.

[8] Saboori H, Hemmati R. Optimal management and planning of storage systems based on particle swarm optimization technique. Journal of Renewable \& Sustainable Energy 2016; 8(2): 453-475.

[9] Chen Z, Li X, Lv B, Jia M. A self-adaptive wireless sensor network coverage method for intrusion tolerance based on particle swarm optimization and cuckoo search. IEEE Trustcom/bigdatase/ispa 2015; 1-10.

[10] Yang P, Miao L, Xue Z, Ye B. Variable neighborhood search heuristic for storage location assignment and storage/retrieval scheduling under shared storage in multishuttle automated storage/retrieval systems. Transportation Research Part E 2015; 79: 164-177.

[11] Joly M, Rocha R, Sousa LCF, Takahashi MT, Mendonça PN, Moraes LAM, Quelhas AD. The strategic importance of teaching Operations Research for achieving high performance in the petroleum refining business. Education for Chemical Engineers 2015; 10: 1-19.

[12] Azaza M, Wallin F. Multi objective particle swarm optimization of hybrid micro-grid system: A case study in Sweden. Energy 2017; 123: 108-118.

[13] Pan I, Das S. Fractional Order AGC for Distributed Energy Resources Using Robust Optimization[J]. IEEE Transactions on Smart Grid 2016; 7(5): 2175-2186.

[14] Mohanasundaram R, Periasamy P S. Clustering Based Optimal Data Storage Strategy Using Hybrid Swarm Intelligence in WSN. Wireless Personal Communications 2015; 85(3): 1381-1397.

\section{Author's information}

Zhongkun Hou (b. 1982) graduated from Southwest Jiaotong University in 2005. He works as a lecturer in Sichuan College of Architectural Technology, China. He has gained the master's degree. He is interested in mechanical automation.

Received December 23, 2019. The final version - February 15, 2020. 